\title{
Using Neural Network Algorithms for Compositional Mapping in STEM EELS
}

\author{
A.Y. Borisevich*, S.V. Kalinin**, A.R. Lupini*, S. Jesse**, H.J. Chang*, M. Huijben***, R. \\ Ramesh****, and S. J. Pennycook* \\ * Materials Science and Technology Division, Oak Ridge National Laboratory, Oak Ridge, TN \\ ** Center for Nanophase Materials Science, Oak Ridge National Laboratory, Oak Ridge, TN \\ ***Faculty of Science \& Technology, University of Twente, Enschede, The Netherlands \\ ****Department of Materials Science, University of California Berkeley, Berkeley, CA
}

By allowing direct imaging of sub-angstrom atomic spacings for the first time, aberration correction has revolutionized electron microscopy. For electron energy loss spectroscopy (EELS) in scanning transmission electron microscopy (STEM), the sensitivity for chemical and electronic structure imaging has improved, enabling single atom detection [1] and mapping of intricate electronic patterns in strongly correlated oxides [2]. Although there are still questions regarding the detailed interpretation at atomic resolution, the necessary data analysis can be performed reliably using characteristic inner-shell ionization edges [3]. In comparison analysis of the low-loss part of EEL spectra, where low-lying core loss edges can overlap strongly with plasmonic excitations, is less straightforward. Here, we demonstrate an approach for compositional mapping across interfaces in STEM-EELS using neural network algorithms.

The EELS data was acquired using a VG Microscopes HB603U operated at $300 \mathrm{kV}$ equipped with a Nion aberration corrector and Gatan Enfina ${ }^{\circledR}$ spectrometer. The EELS compositional mapping at the interfaces was performed using principal component analysis combined with neural network interpolation [4]. Briefly, the 3D spectrum imaging data set in a given energy interval was decomposed into a set of principal components. To perform the mapping, subsets of the spectra far from the interface were identified with specific phases and used as a training set for a linear feed-forward neural network $(5,10,3$ neurons, linear transfer function) trained using back propagation. The trained network was then used to process the full experimental image.

Figure 1 shows the comparison of the mapping results for the $\mathrm{CaTiO}_{3} / \mathrm{SrTiO}_{3}$ interface obtained by (a) conventional background subtraction and integration and (b) using a linear neural network analysis. The results obtained by the two methods are very similar, showing two components and a transition region of a similar (finite) width, thus validating the neural network approach. If a non-linear transfer function is used, the network reconstructs a much sharper boundary between the two components (Fig.1(c)), possibly reflecting the information limit.

The linear neural network approach was then used to analyze a low-loss EELS image of a $\mathrm{SrTiO}_{3} /(\mathrm{La}, \mathrm{Sr}) \mathrm{MnO}_{3} / \mathrm{BiFeO}_{3}$ interface (Fig. 2). Depending on the specific energy range used for the analysis, the resulting compositional maps had a slightly different appearance. It is noteworthy that for the $5-50 \mathrm{eV}$ range, a large region around the $(\mathrm{La}, \mathrm{Sr}) \mathrm{MnO}_{3} / \mathrm{BiFeO}_{3}$ interface was not identified as any of the components, suggesting an anomaly in the dielectric properties. In comparison, similar training in the $70-150 \mathrm{eV}$ interval (which only contains core excitations) does not show this feature. Correspondence with Kramers-Kronig analysis and implications for material properties will be discussed. [5] 


\section{References}

[1] M. Varela, et al., Physical Review Letters 92 (2004).

[2] M. Varela et al., Annu. Rev. Mat. Res., 35 (2005) 539.

[3] R.F. Egerton, EELS in the Electron Microscope (2nd ed.), Plenum, New York (1996).

[4] S. Haykin, Neural Networks: A Comprehensive Foundation, Macmillan (1994)

[5] Research sponsored by the Office of Basic Energy Sciences, Division of Materials Sciences and Engineering, by Laboratory Directed Research and Development (LDRD) funding from Oak Ridge National Laboratory, and by appointment (H.J.C.) to the ORNL Postdoctoral Research Program administered jointly by ORNL and ORISE.
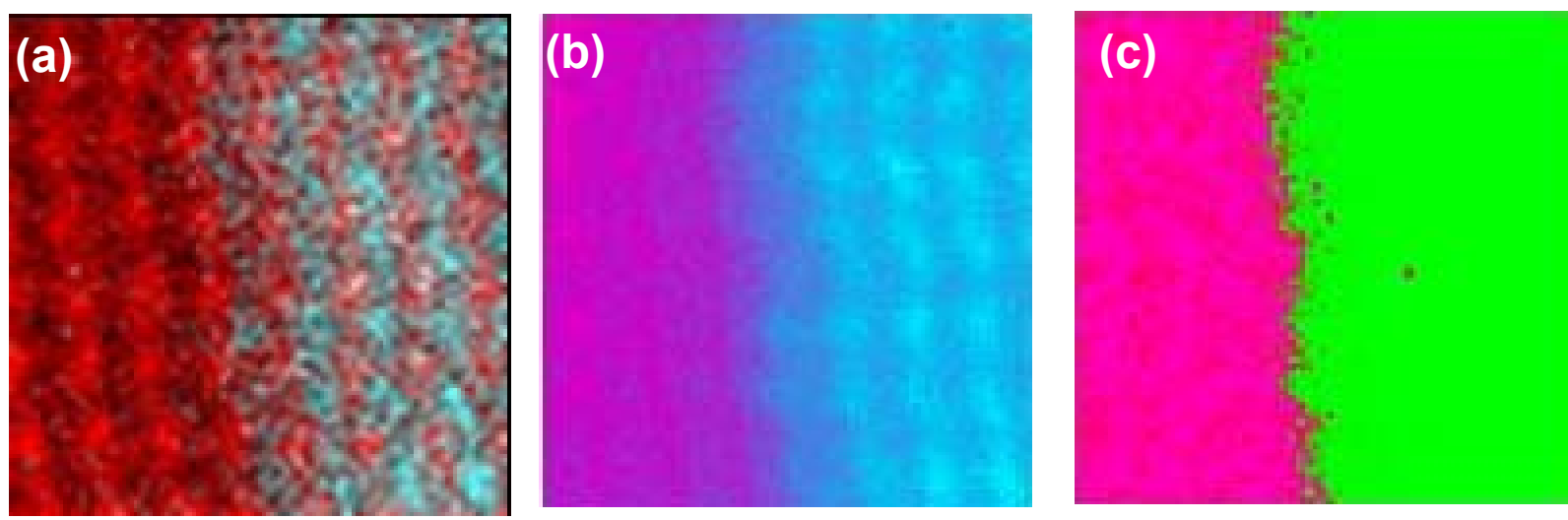

Figure 1. Compositional mapping on $\mathrm{CaTiO}_{3} / \mathrm{SrTiO}_{3}$ interface by (a) background subtraction and integration ( $\mathrm{Ti}$ - red, $\mathrm{Ca}$ - pale blue) (b) linear neural network analysis $\left(\mathrm{SrTiO}_{3}-\right.$ magenta, $\mathrm{CaTiO}_{3}$-blue) (c) non-linear neural network analysis $\left(\mathrm{SrTiO}_{3}-\right.$ magenta, $\mathrm{CaTiO}_{3}$-green).
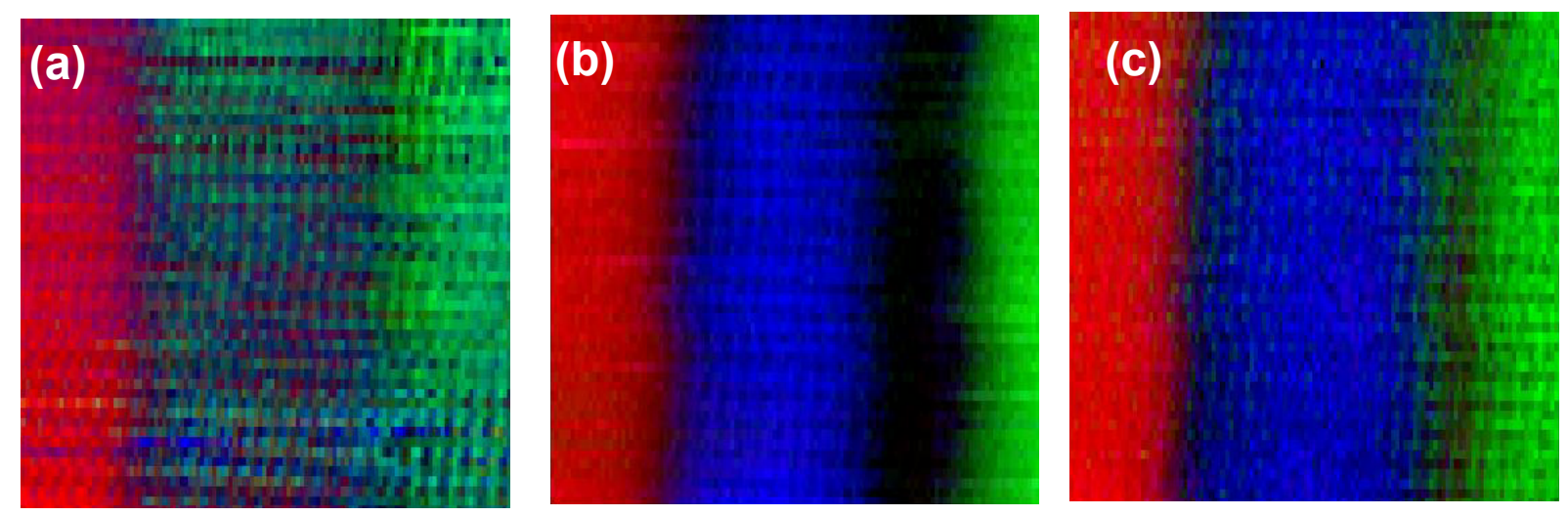

Figure 2. Neural network analysis of a low loss EELS image of $\mathrm{SrTiO}_{3} /(\mathrm{La}, \mathrm{Sr}) \mathrm{MnO}_{3} / \mathrm{BiFeO}_{3}$ interface using (a) zero loss peak (b) energy range of 5 to $50 \mathrm{eV}$ (c) energy range of 70 to 150 $\mathrm{eV}$. 